\title{
High-level expression of a foreign gene by a recombinant baculovirus with an expanded host range
}

\author{
Hye-Seong Kim, Soo-Dong Woo, Woo-Jin Kim, Jae-Young Choi \& Seok-Kwon Kang \\ Division of Applied Biology and Chemistry, College of Agriculture and Life Sciences, Seoul National University, \\ Suwon, 441-744, Korea
}

Received 19 January 1998; accepted 15 July 1999

Key words: baculovirus, expression efficiency, foreign gene, helicase gene, host range

\begin{abstract}
The usefulness of host range expanded viruses as an expression vector system was investigated by following the expression of the E. coli lacZ gene. The host range expanded recombinant viruses were obtained from Sf-21 or BmN-4 cells coinfected with Autographa californica and Bombyx mori nuclear polyhedrosis viruses. Among the host range expanded viruses, RecB-8 and RecS-B6 have similar enzyme digestion profiles but different infection characteristics in cells. Therefore, to study the foreign gene expression efficiency of these two viruses, we constructed recombinant viruses RecB8-LacZ and RecSB6-LacZ containing the lacZ gene instead of the polyhedrin gene. Also, the host range expanded recombinant AcNPV, Bac-BH, containing lacZ gene in the polyhedrin gene locus was constructed by substitution of the $0.6 \mathrm{~kb}$ region within the helicase gene of BacPAK6 with that of BmNPV. $\beta$-Galactosidase expression efficiency by these viruses were determined and compared in Sf-21 and BmN-4 cells. The result showed that Bac-BH has high expression efficiency only in Sf-21 cells, whereas RecB8-LacZ has high expression efficiency both in $\mathrm{Sf}-21$ and $\mathrm{BmN}-4$ cells. Also, in $\mathrm{BmN}-4$ cells, $\beta$-galactosidase expression efficiency of RecB8-LacZ was higher than that of recombinant BmNPV (BmK1-LacZ containing lacZ gene in polyhedrin gene locus). In addition, the expression efficiency was not correlated with virus titer.
\end{abstract}

\section{Introduction}

The insect baculovirus has been used as an efficient vector for the expression of foreign genes in established insect cell lines and whole insects (King and Possee, 1992; Luckow and Summers, 1988, 1989; Maeda, 1989; O'Reilly et al., 1992). It has been shown that baculovirus expression vector systems have several advantages over other expression systems (Maeda, 1989, 1994). Also, recent advances in the molecular biology of baculoviruses and the development of it as an in vitro replication system have revealed some important mechanisms of baculovirus replication. Especially, helicase genes, p35 and pe38 have been shown to be involved in baculovirus host range determination (Albert and Carstens, 1991; Marcel et al., 1994; Pearson et al., 1992). The helicase gene, in addition to being essential for DNA replication, has also been reported as the host range determination factor
(Kondo and Maeda, 1991). In cotransfection experiments of Autographa californica nuclear polyhedrosis virus (AcNPV) and Bombyx mori NPV (BmNPV), the recombinant virus was capable of replicating in both of B. mori and Spodoptera frugiperda cells (Kondo and Maeda, 1991). This was attributed to the $0.6 \mathrm{~kb}$ region of the BmNPV helicase gene (Croizier et al., 1994; Maeda et al., 1993). Although there is a report about the expression of foreign gene using host range expanded virus, they observed only the expression of firefly luciferase gene in both $\mathrm{Sf}$ and $\mathrm{BmN}$ cell lines (Mori et al., 1992).

In this report, we isolated host range expanded recombinants by coinfecting BmNPV and AcNPV in Sf-21 or BmN-4 cells. All of these are able to replicate in Sf-21 cells, BmN-4 cells, larvae of the silkworm, B. mori and the beet armyworm, Spodoptera exigua. Among the recombinant viruses, RecB-8 and RecSB6 have similar enzyme digestion profiles but different 
infection characteristics in cells.

We report here the expression efficiency of the $E$. coli lacZ gene under the control of polyhedrin promoter of these viruses in Sf-21 and BmN-4 cells, and discuss the use of these host range expanded recombinant viruses for potential high-level expression of foreign genes in both cell lines.

\section{Materials and methods}

Viruses

The wild type virus, AcNPV (C6) was kindly provided by Dr. Summers MD (Department of Entomology, Texas A and M University), and BmNPV (K1) was selected from sericultural farms in Korea. The recombinant virus BmK1-LacZ which is recombinant BmNPV containing a $E$. coli lacZ gene instead of polyhedrin gene has been described previously (Woo et al., 1997). The BacPAK6 (Clontech) was used as the recombinant AcNPV containing a lacZ gene in the polyhedrin gene locus. The AcNPV, BmNPV and recombinant viruses were propagated in Sf-21 and BmN-4 cells.

\section{Plasmid and PCR}

Standard plasmid manipulation techniques were used throughout these experiments (Sambrook et al., 1989). In order to clone the helicase $0.6 \mathrm{~kb}$ region, a 16-mer oligonucleotide (forward primer, 5'AAGCTTTGAAACGATG-3') and a 19-mer oligonucleotide (backward primer, 5'-TACTAGCACCGACGAGCTC-3') were synthesized (Croizier et al., 1994; Maeda et al., 1993). For amplification of the polyhedrin gene, a 20-mer oligonucleotide (forward primer, 5'-TAAGTATTTTACTGTTTTCG-3') and a 20-mer oligonucleotide (backward primer, 5' ATTAGTACATTTATTAAGCG-3') were synthesized corresponding to the identical sequences between AcNPV and BmNPV (Hooft van Iddekinge et al., 1983; Iatrou et al., 1985). The amplified PCR products were subcloned into a pGemT PCR cloning vector (Promega) and sequenced by the dideoxynucleotide method (Sanger et al., 1977).

\section{Cell lines and transfection}

Sf-21 and BmN-4 cells were cultured at $27^{\circ} \mathrm{C}$ in TC100 medium (Sigma) supplemented with $10 \%$ fetal bovine serum (Gibco) by the method of Summers and Smith (1987). The transfer vector pBmKSK1LacZ containing lacZ gene instead of polyhedrin gene has been described previously (Woo et al., 1997). Transfection was carried out by a liposomemediated method using transfection reagent, DOTAP (Boehringer Mannheim).

\section{Construction of recombinant baculovirus}

To isolate host range expanded viruses, $\mathrm{Sf}-21$ or $\mathrm{BmN}$ 4 cells were coinfected with 5 M.O.I. of mixture of AcNPV and BmNPV NOVs (non-occluded viruses). After incubation for 5 days at $27{ }^{\circ} \mathrm{C}$, the culture supernatant was subjected to end-point purification using Sf-21 or BmN-4 cells. Following three rounds of end-point purification, 12 recombinant viruses with expanded host range were selected. For the construction of BmNPV helicase gene-mediated recombinant virus with expanded host range, DNA of polyhedronnegative recombinant AcNPV (BacPAK6) was cotransfected with the BmNPV helicase $0.6 \mathrm{~kb}$ cloned plasmid DNA. The transfer vector pBmKSKl-LacZ was cotransfected with DNAs of host range expanded recombinant viruses, RecB-8 and RecS-B6, to produce recombinants containing lacZ gene. Recombinant viruses were isolated by end-point purification.

\section{$\beta$-Galactosidase assay and SDS-PAGE analysis}

The extracellular $\beta$-galactosidase activities were determined at various times ( 0 to 7 days) after infection by recombinant viruses. $\beta$-Galactosidase activity was measured by the $o$-nitrophenyl- $\beta$-D-galactopyranoside (ONPG) assay (Promega). The total protein content of infected cells was analyzed by SDS-PAGE.

\section{Results}

\section{Construction of recombinant viruses}

We isolated 12 recombinant viruses with expanded host range by coinfection with AcNPV and BmNPV. The characteristics of these recombinant viruses were analyzed by restriction enzyme profile and nucleotide sequence. Among these viruses, RecB-8 and RecS-B6 have very similar characteristics. When the restriction enzyme digestion patterns of the RecB-8 and RecS-B6 DMAs were compared with those of parental AcNPV and BmNPV genomic DNAs, the result showed that these viruses were more similar to AcNPV (Figure 1). Also, the $0.6 \mathrm{~kb}$ region within the helicase gene of 

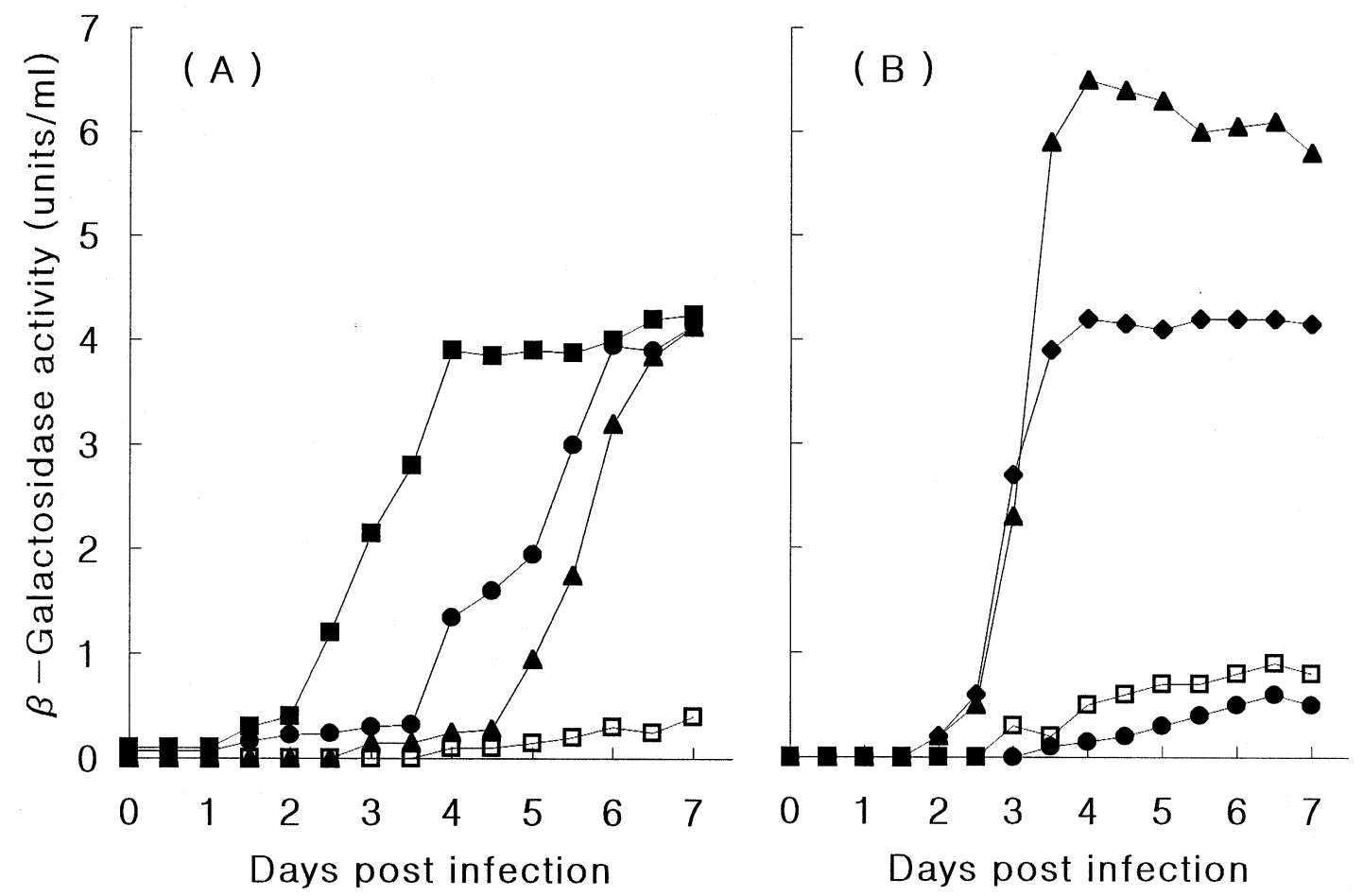

Figure 2. The assay of $\beta$-galactosidase activity in Sf-21 (A) and BmN-4 (B) cells infected with host range expanded recombinant viruses. Cells were infected with 5 MOI of BacPAK6 $(\boldsymbol{\square})$, Bac-BH $(\bullet)$, RecB8-LacZ $(\boldsymbol{\Delta})$, RecSB6-LacZ $(\square)$ or BmK1-LacZ $(\checkmark)$ ). $\beta$-Galactosidase activity was measured by the $o$-nitrophenyl- $\beta$-D-galactopyranoside assay.

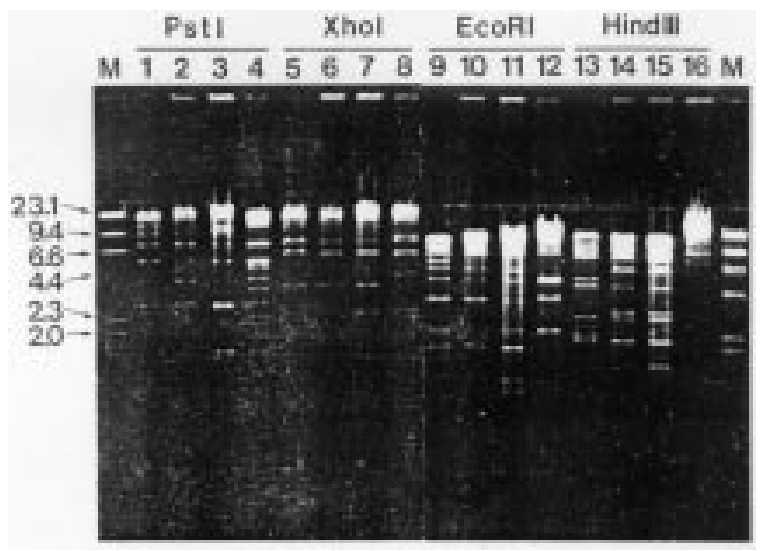

Figure 1. Restriction endonuclease digestion profiles of recombinant and parent virus DNAs. M. Lambda DNA digested with HindIII; Lanes 1, 5, 9 and 13, AcNPV; lanes 2, 6, 10 and 14, RecS-B6; lanes 3, 7, 11 and 15, RecB-8; lanes 4, 8, 12 and 16, BmNPV.

these viruses was identical to that of BmNPV (data not shown). In addition, the polyhedrin gene sequence and its locus of these viruses were identical to that of BmNPV but not AcNPV. To determine the usefulness of these viruses as expression vectors, we reconstructed recombinant viruses RecB8-LacZ and RecSB6-LacZ containing the lacZ gene instead of the polyhedrin

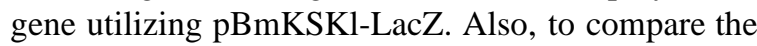
$\beta$-galactosidase expression efficiency of RecB8-LacZ and RecSB6-LacZ in both Sf-21 and BmN-4 cells, we constructed a host range expanded recombinant AcNPV (Bac-BH) by substitution of the $0.6 \mathrm{~kb}$ region within the helicase gene of BacPAK6 with that of BmNPV.

\section{Assay of $\beta$-galactosidase activity produced by recombinant viruses}

$\beta$-Galactosidase activity of BacPAK6 in Sf-21 cells was higher than that produced by other recombinant viruses (Figure $2 \mathrm{~A}$ ). $\beta$-Galactosidase activities by recombinant virus Bac-BH and RecB8-LacZ, were similar to that of BacPAK6 at 7 days post-infection. However, RecSB6-LacZ showed very low $\beta$-galactosidase activity.

In BmN-4 cells, interestingly, $\beta$-galactosidase activity by RecB8-LacZ was higher compared with BmK1-LacZ (Figure 2B). Bac-BH showed very low activity in BmN-4 cells, and RecSB6-LacZ showed similar activity in both of cell lines. 


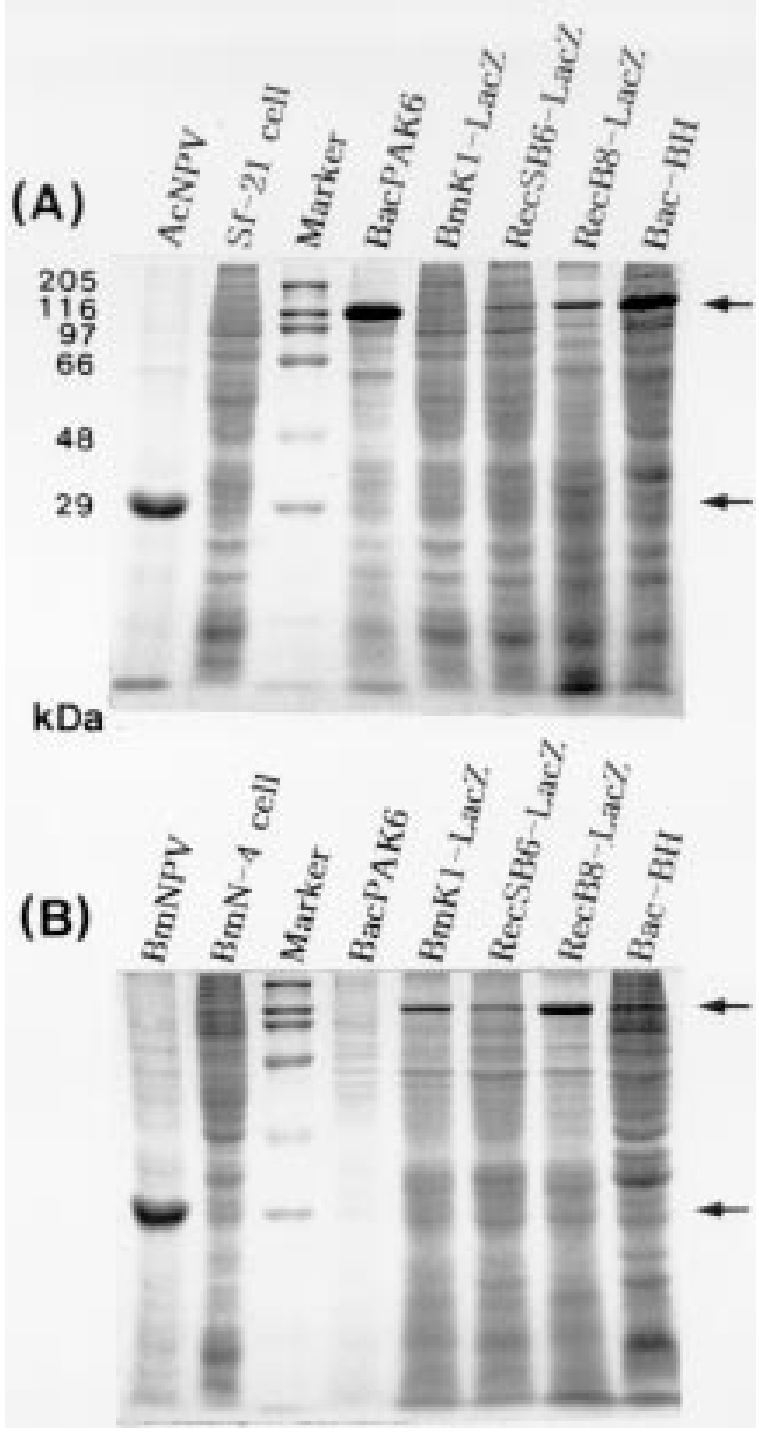

Figure 3. SDS-polyacrylamide gel analysis of $\beta$-galactosidase produced by recombinant viruses in $\mathrm{Sf}-21$ (A) and BmN-4 (B) cells 4 days post-infection. The upper arrows indicate $\beta$-galactosidase protein and lower arrows indicate polyhedrin protein band.

\section{Protein accumulation in cells infected with recombinant viruses}

The protein accumulation of the cells infected with recombinant or wild-type viruses 4 days post-infection were analyzed by SDS-PACE (Figure 3). When cells were infected with wild-type viruses, polyhedra were abundant, but it absent in cells infected with recombinant viruses. The cells infected with recombinant viruses showed a new protein band of approximately $116 \mathrm{kDa}$ corresponding to $\beta$-galactosidase. The density of this $116 \mathrm{kDa}$ protein band reflected the level of

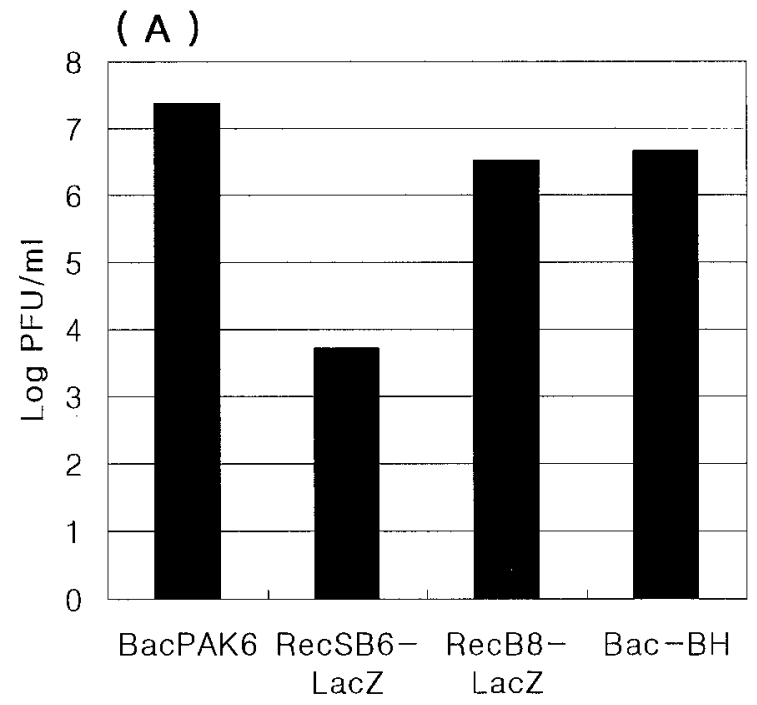

( B )

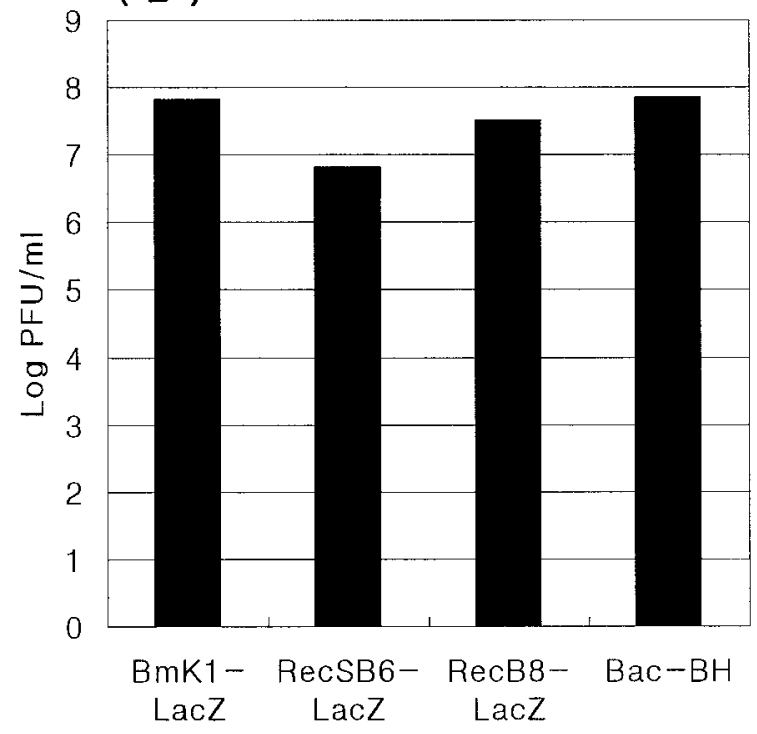

Figure 4. Titration of wild-type and recombinant viruses in $\mathrm{Sf}-21$ (A) and BmN-4 (B) cells at 4 days post-infection.

$\beta$-galactosidase activity produced by the recombinant viruses.

\section{Titration of recombinant viruses}

In order to investigate the reason for the difference of expression efficiency by the recombinant viruses, we determined titers of the recombinant viruses 4 days post-infection (Figure 4). Viral titers of RecB8-LacZ and Bac-BH in Sf-21 cells were almost the same, and these titers were lower than BacPAK6 (Figure 4A). However, expression efficiency of Bac-BH 4 days 
post-infection was about ten-fold higher than that of RecB8-LacZ. Though Bac-BH showed low expression efficiency in $\mathrm{BmN}-4$ cells, its titer was higher than that of BmK1-LacZ and RecB8-LacZ, which showed higher expression efficiency (Figure 4B). Also, the viral titer of RecB8-LacZ, which showed higher activity than BmK1-LacZ, was not higher than that of BmK1-LacZ in BmN-4 cells.

\section{Discussion}

RecB-8 and RecS-B6 have a with wider host range by coinfection of AcNPV and BmNPV and share common characteristics in that they: (i) infect both Sf-21 and BmN-4 cells, (ii) show similar restriction enzyme digestion profiles with AcNPV, (iii) have the $0.6 \mathrm{~kb}$ region of BmNPV helicase gene known to be related to host range determination, and (iv) contain the polyhedrin gene of BmNPV. In order to investigate foreign gene expression under the control of polyhedrin promoter of RecB-8 and RecS-B6 based on these characteristics, we constructed recombinant viruses containing the $E$. coli lacZ gene and examined expression in Sf- 21 and BmN-4 cells. Although post-infection times required to achieve maximum $\beta$-galactosidase activity in Sf-21 cells were variable depending on the virus, recombinant virus BacPAK6, Bac-BH an RecB8-Lacz showed high activity (Figure 2A). However, the level of $\beta$-galactosidase activity produced by RecSB6-LacZ was low through all times after viral infection, despite having almost identical restriction enzyme patterns with RecB8-LacZ. These results indicated that all host range expanded recombinant viruses except RecS-B6 were useful for expression of foreign genes in Sf-21 cells, although the maximum enzyme activities were delayed from 2 to 4 days compared with BacPAK6. Levels of $\beta$-galactosidase in $\mathrm{BmN}-4$ cells infected with recombinant viruses, however, were very different from those in infected Sf-21 cells (Figure 2B). The $\beta$-galactosidase activity produced by $\mathrm{Bac}-\mathrm{BH}$ infection in BmN-4 cells was considerably lower than that in Sf-21 cells. This result showed that Bac-BH is not useful for foreign gene expression in $\mathrm{BmN}-4$ cells. The viral titer of $\mathrm{Bac}-\mathrm{BH}$ in $\mathrm{Sf}-21$ and $\mathrm{BmN}-4$ cells did not show any differences compared with other recombinant viruses (Figure 4). These results indicated that the substituted helicase gene of BmNPV could expand the host range of AcNPV but had a detrimental influence on expression of the very late polyhedrin gene, in $\mathrm{BmN}-4$ cells. Interestingly, one of the random genomic recombinant viruses, RecB-8, showed a different foreign gene expression property. RecB8-LacZ showed a two-fold higher level of $\beta$-galactosidase activity than BmK1-LacZ, a recombinant BmNPV containing the lacZ gene instead of the polyhedrin gene. Foreign gene expression by host range expanded recombinant virus has been previously reported (Mori et al., 1992). The viral titer of RecB8-LacZ in BmN-4 cells was not higher than that of BmK1-LacZ (Figure 4). This indicated that the enhanced expression efficiency of RecB8-LacZ was not due to the difference of virus multiplicity but to some genomic factor influencing the acitivity of very late gene promoter. These results showed that the helicase-mediated host range expanded recombinant virus was able to replicate in both $\mathrm{Sf}-21$ and $\mathrm{BmN}-4$ cells, but was not useful for foreign gene expression in all of them. The host range expanded recombinant viruses obtained by genomic recombination are more useful than only helicase gene-mediated recombinant virus for the expression of foreign genes in both $\mathrm{Sf}-21$ and $\mathrm{BmN}-4$ cells.

Although there is a difference in the foreign gene expression efficiency of RecB8-LacZ and RecSB6$\mathrm{LacZ}$, their genomic structure is very similar to one another. A comparison of the genomic structure of these viruses may explain the reasons for enhanced foreign gene expression of RecB8-LacZ and provide some information concerning late gene expression. We are currently investigating the difference in genomic structure between these two viruses. However, from above results, we conclude that in order to use host range expanded recombinant viruses for foreign gene expression, the host range must be considered as well as other factors influencing very late gene expression.

\section{Acknowledgements}

This study was supported by the Korean Ministry of Education through the Research Fund and grants from the Research Center for New Bio-Materials in Agriculture, Seoul National University.

\section{References}

Albert L \& Carstens EB (1991) Nucleotide sequence of a gene essential for viral DNA replication in the baculovirus Autographa californica nuclear polyhedrosis virus. Virology 181: 336-347.

Croizier G, Croizier L, Argaud O \& Poudevigne D (1994) Extension of Autographa california nuclear polyhedrosis virus host range by interspecific replacement of a short DNA sequence in the p143 helicase gene. Proc Natl Acad Sci USA 91: 48-52. 
Hooft van Iddekinge BJL, Smith GM \& Summers MD (1983) Nucleotide sequence of the polyhedrin gene of Autographa californica nuclear polyhedrosis virus. Virology 131: 561-565.

Iatrou K, Ito K \& Witkiewicz H (1985) Polyhedrin gene of Bombyx mori nuclear polyhedrosis virus. J Virol 54: 436-445.

King LA \& Possee RD (1992) The Baculovirus Expression Vector System. Chapman and Hall, London.

Kondo A \& Maeda S (1991) Host range expansion by recombination of baculoviruses Bombyx mori nuclear polyhedrosis virus and Autographa californica neclear polyhedrosis virus. J Virol 65: 3625-3632.

Luckow VA \& Summers MD (1988) Trends in the development of baculovirus expression vectors. Bio/Technology 6: 47-55.

Luckow VA \& Summers MD (1989) High-level expression of nonfused foreign genes with Autographa californica nuclear polyhedrosis virus expression vectors. Virology 170: 31-39.

Maeda S (1989) Expression of foreign genes in insects using baculovirus vectors. Ann Rev Entomol 34: 351-372.

Maeda S (1994) Expression of foreign genes in insect cells using baculovirus vectors. In: Maramorosch $\mathrm{K}$ and McIntosh AH (eds.) Insect Cell Biotechnology, (pp. 1-31) CRC Press, Boca Raton, FL.

Maeds S, Kamita SG \& Kondo A (1993) Host range expansion of Autographa californica nuclear polyhedrosis virus (NPV) following recombination of a 0.6 kilobase pair DNA fragment originating from Bombyx mori NPV. J Virol 67: 6234-6238.
Marcel K, Ahrens CH, Goldbach RW, Rohrmann GF \& Vlak JM (1994) Identification of genes involved in DNA replication of the Autographa californica baculovirus. Proc Natl Acad Sci USA 91: 11212-11216.

Mori H, Nakazawa H, Shirai N, Shibata N, Sumida M \& Matsubara M (1992) Foreign gene expression by baculovirus vector with an expanded host range. J Gen Virol 73: 1877-1880.

O'Reilly DR, Miller LK \& Luckow VA (1992) Baculovirus Expression Vectors; A Laboratory Manual. W. H. Freeman and Co., New York.

Pearson M, Bjornson R, Pearso G \& Rohrmann G (1992) The Autographa californica baculovirus genome: evidence for multiple replication origins. Science 257: 1382-1384.

Sambrook J, Fritsch EF \& Maniatis T (1989) Molecular Clonings; A Laboratory manual, 2nd ed. Cold Spring Harbor Laboratory Press, Plainview, NY.

Sanger F, Nicklen S \& Coulson AR (1977) DNA sequencing with chain-terminating inhibitors. Proc Natl Acad Sci USA 74: $5463-$ 5467.

Summers MD \& Smith GE (1987) A manual of methods for baculovirus vector and insect cell culture procedures. Texas Agricultural Experiment Station Bulletin No. 1555: 1-56.

Woo SD, Kim WJ, Kim HS, Choi JY, Jin BR \& Kang SK (1997) Effect of silkworm hemolymph on the expression of $E$. coli $\beta$-galactosidase in insect cell lines infected with recombinant baculoviruses. Mol Cells 7: 572-574. 